\title{
Spin-flavor oscillations of Dirac neutrinos in a plane electromagnetic wave
}

\author{
Maxim Dvornikov* \\ Pushkov Institute of Terrestrial Magnetism, Ionosphere and Radiowave Propagation (IZMIRAN), \\ 108840 Moscow, Troitsk, Russia; \\ Physics Faculty, National Research Tomsk State University, 36 Lenin Avenue, 634050 Tomsk, Russia \\ and Nordita, 17 Roslagstullsbacken, 10691 Stockholm, Sweden
}

(Received 17 August 2018; published 29 October 2018)

\begin{abstract}
We study spin and spin-flavor oscillations of Dirac neutrinos in a plane electromagnetic wave with circular polarization. The evolution of massive neutrinos with nonzero magnetic moments in the field of an electromagnetic wave is based on the exact solution of the Dirac-Pauli equation. We formulate the initial condition problem to describe spin-flavor oscillations in an electromagnetic wave. The transition probabilities for spin and spin-flavor oscillations are obtained. In case of spin-flavor oscillations, we analyze the transition and survival probabilities for different neutrino magnetic moments and various channels of neutrino oscillations. As an application of the obtained results, we study the possibility of existence of $\nu_{e \mathrm{~L}} \rightarrow \nu_{\mu \mathrm{R}}$ oscillations in an electromagnetic wave emitted by a highly magnetized neutron star. Our results are compared with findings of other authors.
\end{abstract}

DOI: 10.1103/PhysRevD.98.075025

\section{INTRODUCTION}

The experimental confirmation of oscillations of atmospheric and solar neutrinos in Refs. [1,2] is the direct indication that neutrinos have nonzero masses and mixing, which, in its turn, unambiguously points to the physics beyond the standard model. This experimental success was followed by the determination of other parameters in the neutrino mixing matrix, including $\theta_{13}$ (see, e.g., Ref. [3]) and the $C P$ violation phase (see, e.g., Ref. [4]).

Despite the great importance of neutrino flavor oscillations for the experimental studies of properties of these particles, other channels of neutrino oscillations are of interest for the evolution of astrophysical and cosmological neutrinos [5]. In the present work, we deal mainly with spin-flavor oscillations of neutrinos, which imply the conversion of the type $\nu_{\beta \mathrm{L}} \rightarrow \nu_{\alpha \mathrm{R}}$, where both flavor, $\alpha, \beta=e, \mu, \tau, \ldots$, and helicity, $\mathrm{L}, \mathrm{R}$, change. This type of neutrino transition implies that these particles possess nonzero magnetic moments and interact with a strong electromagnetic field. Neutrino electromagnetic properties are reviewed in Ref. [6], pages 461-479.

The majority of studies of neutrino spin-flavor oscillations involve the neutrino interaction with a constant

\footnotetext{
*maxdvo@izmiran.ru
}

Published by the American Physical Society under the terms of the Creative Commons Attribution 4.0 International license. Further distribution of this work must maintain attribution to the author(s) and the published article's title, journal citation, and DOI. Funded by SCOAP . transverse magnetic field. Other configurations of electromagnetic fields, including an electromagnetic wave, were used in Refs. [7,8] in the examination of spin-flavor oscillations. The neutrino interaction with an electromagnetic wave is important for the studies of neutrino propagation in strong laser pulses $[9,10]$.

In the present work, we study neutrino spin and spinflavor oscillations in a plane electromagnetic wave on the basis of the exact solution of the Dirac-Pauli equation for a massive neutrino in this external electromagnetic field [11]. In our analysis, we suggest that neutrinos are Dirac particles. Although some theoretical models of the neutrino mass generation point out that neutrinos are likely to be Majorana particles [ [6] pp. 387-460], the issue of the neutrino nature is still open [12].

Our work is organized as follows. In Sec. II, we start with the studies of neutrino spin oscillations in a plane electromagnetic wave with circular polarization within one neutrino mass eigenstate. Then, in Sec. III, we generalize our treatment to account for neutrino spin-flavor oscillations with the great transition magnetic moment. The description of spin-flavor oscillations is based on the formulation of the initial condition problem for flavor neutrinos [13]. The possibility of an astrophysical application is also considered in Sec. III. The influence of the small diagonal magnetic moments on spin-flavor oscillations is studied in Sec. IV. Spin-flavor oscillations of neutrinos with great diagonal magnetic moments are considered in Sec. V. We summarize our results in Sec. VI. The matrix elements of the neutrino spin interaction are calculated in Appendix. 


\section{SPIN OSCILLATIONS IN AN ELECTROMAGNETIC WAVE}

In this section, we consider neutrino spin oscillations, within one neutrino generation, in a plane electromagnetic wave with the circular polarization. Our analysis is based on the exact solution of the Dirac-Pauli equation for a massive neutral fermion found in Ref. [11].

We take that a neutrino is a Dirac particle. In this section, we neglect the mixing between different neutrino generations. Assuming that the considered neutrino mass eigenstate has the nonzero mass $m$ and the magnetic moment $\mu$, the Dirac equation for such a neutrino, described by the bispinor $\psi$, in the external electromagnetic field $F_{\mu \nu}=$ $\partial_{\mu} A_{\nu}-\partial_{\nu} A_{\mu}=(\mathbf{E}, \mathbf{B})$ reads

$$
\left[\mathrm{i} \gamma^{\mu} \partial_{\mu}-m-\frac{\mu}{2} \sigma_{\mu \nu} F^{\mu \nu}\right] \psi=0,
$$

where $\gamma^{\mu}=\left(\gamma^{0}, \gamma\right)$ and $\sigma_{\mu \nu}=\frac{\mathrm{i}}{2}\left[\gamma_{\mu}, \gamma_{\nu}\right]_{-}$are the Dirac matrices. In the following, we use the standard representation for the Dirac matrices [14].

We take that the external electromagnetic field is in the form of a plane electromagnetic wave propagating in the positive direction of the $z$ axis. It is convenient to choose the following gauge for the vector potential: $A^{\mu}=(0, \mathbf{A})$. Neglecting the dispersion of the wave, we can take that $\mathbf{A}=\mathbf{A}(t-z)$. The electric and magnetic fields are $\mathbf{E}=$ $-\mathbf{A}^{\prime}$ and $\mathbf{B}=\left(\mathbf{e}_{z} \times \mathbf{E}\right)$, where the prime means the derivative with respect to the whole argument of $\mathbf{A}$.

The exact solution of Eq. (2.1) was found in Ref. [11] for the arbitrary propagation of the fermion with respect to the electromagnetic wave. In the present work, we consider a special situation when a neutrino propagates along the wave, i.e., $\psi=\psi(z, t)$. In this case, the solution of Eq. (2.1) has the form

$$
\psi=\frac{e^{-\mathrm{i} E t+\mathrm{i} p z}}{2 \sqrt{E(E-p)}}\left(\begin{array}{c}
{[m+E-p] v} \\
{[m-E+p] \sigma_{3} v}
\end{array}\right),
$$

where $v=v(t-z)$ is the two component spinor, $E=$ $\sqrt{p^{2}+m^{2}}$ is the neutrino energy, $p$ is the neutrino momentum, and $\boldsymbol{\sigma}=\left(\sigma_{1}, \sigma_{2}, \sigma_{3}\right)$ are the Pauli matrices. It is interesting to notice that $|\psi|^{2}=1$ if $|v|^{2}=1$. The motivation to study neutrinos propagating along the electromagnetic wave results from Fig. 1.

The spinor $v$ was found in Ref. [11] to obey the equation

$$
\mathrm{i} v^{\prime}=-\mu(\boldsymbol{\sigma} \mathbf{B}) v \text {. }
$$

To proceed with the analysis of the solution of Eq. (2.3) we suppose that we have a circularly polarized electromagnetic wave, i.e., $B_{x}=B_{0} \cos [\omega(t-z)], B_{y}=B_{0} \sin [\omega(t-z)]$, and $B_{z}=0$, where $B_{0}$ is the amplitude of the wave and $\omega$ is its frequency. In this case, the solution of Eq. (2.3) has the form

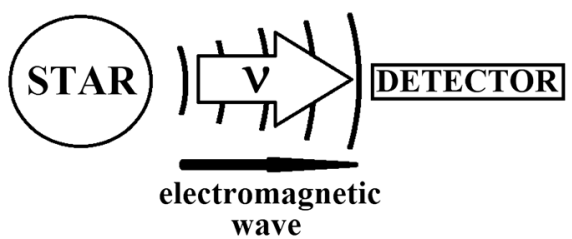

FIG. 1. The schematic illustration of the neutrino propagation in an electromagnetic wave. If both a neutrino and a wave are produced by the same source, e.g., a neutron star (NS; see Sec. III), they propagate collinearly towards a detector. Thus one studies oscillations of neutrinos, created in a star, under the influence of an electromagnetic wave emitted by the same star. It is the motivation to use the neutrino wave function in Eq. (2.2).

$$
\begin{aligned}
v(\phi)= & \exp \left(-\mathrm{i} \sigma_{3} \omega \phi / 2\right)\left\{\cos \left[\Omega\left(\phi-\phi_{0}\right)\right]\right. \\
& \left.+\frac{\mathrm{i}}{\Omega}\left[\mu B_{0} \sigma_{1}+\omega \sigma_{3} / 2\right] \sin \left[\Omega\left(\phi-\phi_{0}\right)\right]\right\} \\
& \times \exp \left(\mathrm{i} \sigma_{3} \omega \phi_{0} / 2\right) v_{0},
\end{aligned}
$$

where $\phi=t-z$ is the current phase, $\phi_{0}=t_{0}-z_{0}$ is the initial phase, $v_{0}=v\left(\phi_{0}\right)$ is the initial spinor, and $\Omega=$ $\sqrt{\left(\mu B_{0}\right)^{2}+\omega^{2} / 4}$. One can see in Eq. (2.4) that $v=v_{0}$ if $\phi=\phi_{0}$. Moreover $|v|^{2}=\left|v_{0}\right|^{2}$.

Now we should specify the initial condition. Let us suppose that $v_{0}^{\mathrm{T}}=(0,1)$. In this case, using Eq. (2.2), one can check that $P_{-} \psi_{0}=\psi_{0}$, where $\psi_{0}$ is the initial bispinor, corresponding to $v=v_{0}, P_{ \pm}=\left(1 \pm \Sigma_{z}\right) / 2$ are the helicity projection operators, and $\boldsymbol{\Sigma}=\gamma^{5} \gamma^{0} \gamma$ and $\gamma^{5}=\mathrm{i} \gamma^{0} \gamma^{1} \gamma^{2} \gamma^{3}$ are the Dirac matrices. It means that initially a neutrino is lefthanded, i.e., its spin is opposite to the particle momentum.

Neutrino spin oscillations correspond to the appearance of the nonzero component $\psi_{+}$, i.e., the right-handed polarization, at $\phi>\phi_{0}$, where $\psi_{+}=P_{+} \psi$. The probability of $\mathrm{L} \rightarrow \mathrm{R}$ transitions is $P_{\mathrm{L} \rightarrow \mathrm{R}}=\psi^{\dagger} P_{+} \psi$. Using Eq. (2.2), one can show that $P_{\mathrm{L} \rightarrow \mathrm{R}}=\left(1+v^{\dagger} \sigma_{3} v\right) / 2$; i.e., the transition probability is completely defined by the evolution of the neutrino spin in Eq. (2.3).

After lengthy but straightforward calculations based on Eq. (2.4), we can represent $P_{\mathrm{L} \rightarrow \mathrm{R}}$ in the form

$P_{\mathrm{L} \rightarrow \mathrm{R}}=\frac{\mu^{2} B_{0}^{2}}{\mu^{2} B_{0}^{2}+\omega^{2} / 4} \sin ^{2}\left[\sqrt{\mu^{2} B_{0}^{2}+\omega^{2} / 4}\left(\phi-\phi_{0}\right)\right]$.

One can see in Eq. (2.5) that the transition probability is a function of $\Delta \phi=\phi-\phi_{0}$; i.e., it depends on both $t$ and $z$. Thus neutrino spin oscillations can happen both in time and in space. It is owing to the fact that the spinor $v$ in Eqs. (2.2) and (2.4) depends on $\phi$. It is the feature of the field theory based approach for the description of neutrino oscillations.

The coordinate dependence of $P_{\mathrm{L} \rightarrow \mathrm{R}}$ in Eq. (2.5) becomes important if a neutrino interacts with an electromagnetic wave for a quite long time. In such a situation, the particle initial wave packet becomes broad enough. It can 
happen for relatively low energy neutrinos. In practice, a neutrino is an ultrarelativistic particle. We can localize it and attribute a mean velocity $\beta=p / E$ of its wave packet. Thus $z=\beta t$ and $z_{0}=\beta t_{0}$.

In this way to modify the coordinate dependence of $P_{\mathrm{L} \rightarrow \mathrm{R}}$ in Eq. (2.5), we rederive the transition probability of neutrino spin oscillations in a circularly polarized electromagnetic wave obtained in Ref. [7] on the basis of the quasiclassical approach. For this purpose one should set $\Delta m^{2}=0$ and neglect the interaction with matter in the corresponding expressions in Ref. [7] since, in this section, we study neutrino spin oscillations induced by the electromagnetic wave only within one neutrino generation. In the following section, we generalize this formalism to describe neutrino spin-flavor oscillations.

\section{SPIN-FLAVOR OSCILLATIONS: GREAT TRANSITION MAGNETIC MOMENT}

In this section, we extend the formalism developed in Sec. II to describe neutrino spin-flavor oscillations. For this purpose we discuss the system of two flavor neutrinos and formulate the initial condition problem for these particles. Here we focus on the case of the great transition magnetic moment.

For simplicity, we consider the system of two flavor neutrinos $\left(\nu_{\alpha}, \nu_{\beta}\right)$. The generalization of the formalism for a greater number of neutrino flavors is straightforward. For instance, we can take that $\nu_{\beta}=\nu_{e}$ and $\nu_{\alpha}=\nu_{\mu, \tau}$. The Lagrangian for this system in the presence of the external electromagnetic field reads

$\mathcal{L}=\sum_{\lambda=\alpha, \beta} \bar{\nu}_{\lambda} i \gamma^{\mu} \partial_{\mu} \nu_{\lambda}-\sum_{\lambda, \lambda^{\prime}=\alpha, \beta} \bar{\nu}_{\lambda}\left(m_{\lambda \lambda^{\prime}}+\frac{1}{2} M_{\lambda \lambda^{\prime}} \sigma_{\mu \nu} F^{\mu \nu}\right) \nu_{\lambda^{\prime}}$,

where $\left(m_{\lambda \lambda^{\prime}}\right)$ and $\left(M_{\lambda \lambda^{\prime}}\right)$ are the matrices of masses and magnetic moments in the flavor eigenstates basis. As in Sec. II, we suppose that only an electromagnetic wave, propagating along the $z$ axis, is present in the system and neutrinos move in the direction of the wave.

We define the initial conditions in the system described by the Lagrangian in Eq. (3.1). For this purpose, we suppose that initially $\nu_{\alpha}(z, t=0)=0$ and $\nu_{\beta}(z, t=0)=f \nu_{i \mathrm{~L}}$, where $f=f(z)$ is the given coordinate dependence of the initial wave function and $\nu_{i \mathrm{~L}}$ is a constant bispinor. Let us suppose that $\Sigma_{z} \nu_{i \mathrm{~L}}=-\nu_{i \mathrm{~L}}$. For example, we can take $\nu_{i \mathrm{~L}}^{\mathrm{T}}=$ $(1 / \sqrt{2})(0,1,0,-1)$. Such a choice of the initial wave functions corresponds to the presence of left-handed electron neutrinos (if $\nu_{\beta} \equiv \nu_{e}$ ) and the absence of neutrinos of other flavors.

If only two neutrino flavors are present in the system, we can introduce the neutrino mass eigenstates $\psi_{a}, a=1,2$, to diagonalize the mass matrix in Eq. (3.1). The corresponding matrix transformation reads $\nu_{\lambda}=\sum_{a=1,2} U_{\lambda a} \psi_{a}, \quad\left(U_{\lambda a}\right)=\left(\begin{array}{cc}\cos \theta & -\sin \theta \\ \sin \theta & \cos \theta\end{array}\right)$,

where $\theta$ is the vacuum mixing angle. Note that we can say whether neutrinos are Dirac or Majorana only after the transition to the mass eigenstates basis in Eq. (3.2).

The Dirac equation for $\psi_{a}$ has the form

$\mathrm{i} \dot{\psi}_{a}=H_{a} \psi_{a}+V \psi_{b}, \quad a, b=1,2, \quad a \neq b$,

where

$$
\begin{aligned}
H_{a} & =(\boldsymbol{\alpha} \mathbf{p})+\beta m_{a}-\mu_{a} \beta[(\boldsymbol{\Sigma} \mathbf{B})-\mathrm{i}(\boldsymbol{\alpha} \mathbf{E})], \\
V & =-\mu \beta[(\boldsymbol{\Sigma} \mathbf{B})-\mathrm{i}(\boldsymbol{\alpha} \mathbf{E})] .
\end{aligned}
$$

Here $\mu_{a} \equiv\left(\mu_{11}, \mu_{22}\right)$ and $\mu \equiv \mu_{12}$ are the diagonal and transition magnetic moments in the mass eigenstates basis, $\left(\mu_{a b}\right)=U^{\dagger} M U$, and $\alpha=\gamma^{0} \gamma$ and $\beta=\gamma^{0}$ are the Dirac matrices.

First, we discuss the situation when $\mu_{a} \ll \mu$. If we neglect the antiparticle degrees of freedom, the general solution of Eq. (3.3) has the form

$$
\begin{aligned}
\psi_{a}(z, t) & =\int_{-\infty}^{+\infty} \frac{\mathrm{d} p}{2 \pi} \sum_{s= \pm} a_{a s} e^{-\mathrm{i} E_{a} t+\mathrm{i} p z} u_{a s}, \\
u_{a s} & =\frac{1}{2 \sqrt{E_{a}\left(E_{a}-p\right)}}\left(\begin{array}{c}
{\left[m_{a}+E_{a}-p\right] v_{s}} \\
{\left[m_{a}-E_{a}+p\right] \sigma_{3} v_{s}}
\end{array}\right),
\end{aligned}
$$

where $E_{a}=\sqrt{p^{2}+m_{a}^{2}}$ are the energies of different mass eigenstates, $v_{+}^{\mathrm{T}}=(1,0)$ corresponds to a right-handed neutrino, and $v_{-}^{\mathrm{T}}=(0,1)$ corresponds to a left-handed particle since $\sigma_{3} v_{ \pm}= \pm v_{ \pm}$. Note that we omit the index $a$ in the spinors $v_{s}$ since we neglect $\mu_{a}$. We also mention the orthogonality of the basis bispinors, $u_{a \pm}^{\dagger} u_{a \mp}=0$.

The coefficients $a_{a s}$ are supposed to be $c$-number functions rather than operators acting on Fock states. If we assume that they depend on neither $t$ nor $z$, the wave functions in Eq. (3.5) would satisfy the equations $\mathrm{i} \dot{\psi}_{a}=H_{a} \psi_{a}$. To account for the potential $V$ in Eq. (3.4), which mixes different mass eigenstates in Eq. (3.3), we have to suppose that $a_{a s}$ are no longer constant. Such an approach for the description of the evolution of neutrino mass eigenstates in the presence of external fields was used in Ref. [13]. However, unlike Ref. [13], where the corresponding coefficients depend on time only, here we suppose that $a_{a s}=a_{a s}(t-z)$. Our main goal is to study the behavior of these coefficients.

Substituting the ansatz in Eq. (3.5) to Eq. (3.3), we obtain the equation for $a_{a s}$, 
$\mathrm{i} \sum_{s= \pm} a_{a s}^{\prime} u_{a s^{\prime}}^{\dagger}\left(1-\alpha_{z}\right) u_{a s}=\sum_{s= \pm} u_{a s^{\prime}}^{\dagger} V u_{2 s} e^{\mathrm{i}\left(E_{a}-E_{b}\right) t} a_{b s}, \quad a \neq b$.

Accounting for the mean values

$$
\begin{aligned}
u_{a s^{\prime}}^{\dagger}\left(1-\alpha_{z}\right) u_{a s} & =\left(1-\frac{p}{E_{a}}\right) \delta_{s s^{\prime}}, \\
u_{1 s^{\prime}}^{\dagger} V u_{2 s} & =-\mu v_{s^{\prime}}^{\dagger}(\boldsymbol{\sigma} \mathbf{B}) v_{s} \sqrt{\frac{\left(E_{1}-p\right)\left(E_{2}-p\right)}{E_{1} E_{2}}}, \\
v_{ \pm}^{\dagger}(\boldsymbol{\sigma} \mathbf{B}) v_{\mp} & =B_{0} \exp [\mp i \omega(t-z)],
\end{aligned}
$$

we can rewrite Eq. (3.6) in the form

$$
\begin{aligned}
\mathrm{i} a_{a \pm}^{\prime}= & -\mu B_{0} \sqrt{\frac{E_{a}\left(E_{b}-p\right)}{E_{b}\left(E_{a}-p\right)}} \\
& \times \exp \left[\mathrm{i}\left(E_{a}-E_{b}\right) t \mp \mathrm{i} \omega(t-z)\right] a_{b \mp} .
\end{aligned}
$$

In Eqs. (3.7) and (3.8), we suppose that one deals with a circularly polarized wave.
Before we proceed with the analysis of the evolution of $a_{a s}$ we should specify the initial conditions for them. As in Sec. II, we suppose that neutrinos are localized along their trajectories. However, since different mass eigenstates have different masses, we assume that $z=\bar{\beta}$, where $\bar{\beta}$ is the center of inertia velocity [15], $\bar{\beta}=2 p /\left(E_{1}+E_{2}\right)$. If neutrinos are ultrarelativistic, one gets that $\bar{\beta} \approx$ $1-\left(m_{1}^{2}+m_{2}^{2}\right) / 4 p^{2}$. Therefore, if $t=0, a_{a s}$ should be taken at zero argument: $a_{a s}(0)$.

Accounting for the initial condition for the flavor eigenstates specified above, we obtain that

$$
\begin{aligned}
& a_{1-}(0)=\sin \theta f(p)\left(u_{1-}^{\dagger} \nu_{i \mathrm{~L}}\right), \quad f(p)=\int_{-\infty}^{+\infty} f(z) e^{-\mathrm{i} p z} \mathrm{~d} z \\
& a_{2-}(0)=\cos \theta f(p)\left(u_{2-}^{\dagger} \nu_{i \mathrm{~L}}\right),
\end{aligned}
$$

and $a_{a+}(0)=0$. Taking into account the explicit form of the initial bispinor $\nu_{i \mathrm{~L}}$ and $u_{a-}$ in Eq. (3.5), we get that $\left(u_{a-}^{\dagger} \nu_{i \mathrm{~L}}\right)=m_{a} / \sqrt{2 E_{a}\left(E_{a}-p\right)}$. If neutrinos are ultrarelativistic, $\left(u_{a-}^{\dagger} \nu_{i \mathrm{~L}}\right) \rightarrow 1$.

Taking into account the dependence of $a_{a s}$ on $t$ in Eq. (3.9), $a_{a s}=a_{a s}(t[1-\bar{\beta}])$, we can rewrite Eq. (3.9) as the effective Schrödinger equation,

$$
\begin{aligned}
& \mathrm{i} \frac{\mathrm{d}}{\mathrm{d} t} \Psi=H_{\text {eff }} \Psi \\
& H_{\mathrm{eff}}=-\mu B_{0}(1-\bar{\beta})\left(\begin{array}{cccc}
0 & 0 & 0 & \xi \exp \left(\mathrm{i} \Phi_{-} t\right) \\
0 & 0 & \xi \exp \left(\mathrm{i} \Phi_{+} t\right) & 0 \\
0 & \exp \left(-\mathrm{i} \Phi_{+} t\right) / \xi & 0 & 0 \\
\exp \left(-\mathrm{i} \Phi_{-} t\right) / \xi & 0 & 0 & 0
\end{array}\right),
\end{aligned}
$$

where $\Psi^{\mathrm{T}}=\left(a_{1+}, a_{1-}, a_{2+}, a_{2-}\right)$ and

$$
\begin{aligned}
\Phi_{ \pm} & =E_{1}-E_{2} \pm \omega(1-\bar{\beta}) \\
& \approx \frac{m_{1}^{2}-m_{2}^{2}}{2 p} \pm \omega \frac{m_{1}^{2}+m_{2}^{2}}{4 p^{2}}, \\
\xi & =\sqrt{\frac{E_{1}\left(E_{2}-p\right)}{E_{2}\left(E_{1}-p\right)} \approx \frac{m_{2}}{m_{1}} .}
\end{aligned}
$$

In Eqs. (3.12) and (3.13), we use the approximation of ultrarelativistic neutrinos.

One can see that $H_{\text {eff }}$ in Eq. (3.11) is non-Hermitian since $\xi \neq 1$ because $m_{1} \neq m_{2}$. This fact results from the assumption that both mass eigenstates with different masses propagate with the same velocity $\bar{\beta}$. Nevertheless we can

approximately set $\xi=1$. Indeed, let us assume that $m_{a} \sim$ $1 \mathrm{eV}$ [16]. Below, we are interested in the $\nu_{e} \nu_{\mu}$-oscillations channel. In this situation, $\delta m^{2}=m_{1}^{2}-m_{2}^{2} \approx 7.6 \times 10^{-5} \mathrm{eV}^{2}$ [17]. Therefore, $\left|m_{1}-m_{2}\right| / m_{a} \sim 10^{-2}$. Hence, we can take that $\xi=1$ with a sufficient level of accuracy. Note that we should keep $\delta m^{2} \neq 0$ in $\Phi_{ \pm}$in Eq. (3.12) since these terms contribute to the phase of neutrino oscillations, which is very sensitive to the change of parameters.

Let us change the variables in Eq. (3.11),

$$
\begin{aligned}
& \Psi=\mathcal{U} \tilde{\Psi}, \\
& \mathcal{U}=\operatorname{diag}\left(e^{i \Phi_{-} t / 2}, e^{i \Phi_{+} t / 2}, e^{-i \Phi_{+} t / 2}, e^{-i \Phi_{-} t / 2}\right) .
\end{aligned}
$$

The wave function $\tilde{\Psi}^{\mathrm{T}}=\left(\tilde{a}_{1+}, \tilde{a}_{1-}, \tilde{a}_{2+}, \tilde{a}_{2-}\right)$ obeys the modified Schrödinger equation, 


$$
\begin{aligned}
\mathrm{i} \frac{\mathrm{d}}{\mathrm{d} t} \tilde{\Psi} & =\tilde{H}_{\text {eff }} \tilde{\Psi}, \\
\tilde{H}_{\text {eff }} & =\left(\begin{array}{cccc}
\Phi_{-} / 2 & 0 & 0 & -\mu B_{0}(1-\bar{\beta}) \\
0 & \Phi_{+} / 2 & -\mu B_{0}(1-\bar{\beta}) & 0 \\
0 & -\mu B_{0}(1-\bar{\beta}) & -\Phi_{+} / 2 & 0 \\
-\mu B_{0}(1-\bar{\beta}) & 0 & 0 & -\Phi_{-} / 2
\end{array}\right) .
\end{aligned}
$$

We recall that we set $\xi=1$ in the $\mu B_{0}$-terms in Eq. (3.15).

The solution of Eq. (3.15) has the form

$$
\tilde{\Psi}(t)=\sum_{\zeta= \pm}\left[e^{-i \Omega_{\zeta} t}\left(U_{\zeta} \otimes U_{\zeta}^{\dagger}\right)+e^{i \Omega_{\zeta} t}\left(V_{\zeta} \otimes V_{\zeta}^{\dagger}\right)\right] \tilde{\Psi}_{0}
$$

where $\Omega_{ \pm}=\sqrt{\mu^{2} B_{0}^{2}(1-\bar{\beta})^{2}+\Phi_{ \pm}^{2} / 4}$ and

$$
\begin{gathered}
U_{+}=\frac{\sqrt{\Omega_{+}+\Phi_{+} / 2}}{\sqrt{2 \Omega_{+}}}\left(\begin{array}{c}
0 \\
1 \\
-\frac{\mu B_{0}(1-\bar{\beta})}{\Omega_{+}+\Phi_{+} / 2} \\
0
\end{array}\right), \\
V_{+}=\frac{\sqrt{\Omega_{+}+\Phi_{+} / 2}}{\sqrt{2 \Omega_{+}}}\left(\begin{array}{c}
\mu B_{0}(1-\bar{\beta}) \\
\Omega_{+}+\Phi_{+} / 2 \\
1 \\
0
\end{array}\right), \\
U_{-}=\frac{\sqrt{\Omega_{-}+\Phi_{-} / 2}}{\sqrt{2 \Omega_{-}}}\left(\begin{array}{c}
1 \\
0 \\
0 \\
-\frac{\mu B_{0}(1-\bar{\beta})}{\Omega_{-}+\Phi_{-} / 2}
\end{array}\right), \\
V_{-}=\frac{\sqrt{\Omega_{-}+\Phi_{-} / 2}}{\sqrt{2 \Omega_{-}}}\left(\begin{array}{c}
\Omega_{-}+\Phi_{-} / 2 \\
0 \\
0 \\
1
\end{array}\right),
\end{gathered}
$$

are the eigenvectors of $\tilde{H}_{\text {eff }}: \quad \tilde{H}_{\text {eff }} U_{\zeta}=\Omega_{\zeta} U_{\zeta}$ and $\tilde{H}_{\text {eff }} V_{\zeta}=-\Omega_{\zeta} V_{\zeta}$.

Using Eqs. (3.10), (3.16), and (3.17), we find that the coefficients $a_{a+}(t)$ have the form

$$
\begin{aligned}
& a_{1+}(t)=\mathrm{i} e^{\mathrm{i} \Phi_{-} t / 2}(1-\bar{\beta}) \frac{\mu B_{0}}{\Omega_{-}} \sin \left(\Omega_{-} t\right) f(p) \cos \theta, \\
& a_{2+}(t)=\mathrm{i} e^{-\mathrm{i} \Phi_{+} t / 2}(1-\bar{\beta}) \frac{\mu B_{0}}{\Omega_{+}} \sin \left(\Omega_{+} t\right) f(p) \sin \theta .
\end{aligned}
$$

The explicit form of $a_{a_{-}}(t)$ is not important for our purposes since these coefficients do not contribute to the evolution of the right-handed neutrino states.
We are interested in the appearance of right-handed neutrinos of the flavor $\alpha$ in a beam initially consisting of $\nu_{\beta \mathrm{L}}$. We suppose that the initial wave packet is quite wide; i.e., we take that $f(p)=2 \pi \delta\left(p-p_{0}\right)$, where $p_{0}$ is the initial momentum. In the following, we omit the subscript 0 for brevity. Using Eqs. (3.2), (3.5), and (3.18), we obtain that the wave function $\nu_{\alpha \mathrm{R}}$ has the form

$$
\begin{aligned}
\nu_{\alpha \mathrm{R}}(z, t)= & \int_{-\infty}^{+\infty} \frac{\mathrm{d} p}{2 \pi}\left[\cos \theta e^{-\mathrm{i} E_{1} t} a_{1+}(t) u_{1+}\right. \\
& \left.-\sin \theta e^{-\mathrm{i} E_{2} t} a_{2+}(t) u_{2+}\right] \\
= & {\left[\cos \theta e^{-\mathrm{i} E_{1} t} a_{1+}(t)\left(\nu_{f \mathrm{R}}^{\dagger} u_{1+}\right)\right.} \\
& \left.-\sin \theta e^{-\mathrm{i} E_{2} t} a_{2+}(t)\left(\nu_{f \mathrm{R}}^{\dagger} u_{2+}\right)\right] \nu_{f \mathrm{R}},
\end{aligned}
$$

where $\nu_{f \mathrm{R}}^{\mathrm{T}}=(1 / \sqrt{2})(1,0,1,0)$. Basing on Eq. (3.5), one gets that $\left(\nu_{f \mathrm{R}}^{\dagger} u_{a+}\right)=m_{a}\left[2 E_{a}\left(E_{a}-p\right)\right]^{-1 / 2}$. If we study ultrarelativistic neutrinos, then $\left(\nu_{f \mathrm{R}}^{\dagger} u_{a+}\right) \rightarrow 1$.

Using Eqs. (3.18) and (3.19), as well as considering ultrarelativistic neutrinos, we obtain the transition probability for $\nu_{\beta \mathrm{L}} \rightarrow \nu_{\alpha \mathrm{R}}$ oscillations,

$$
\begin{aligned}
& P_{\nu_{\beta \mathrm{L}} \rightarrow \nu_{\alpha \mathrm{R}}}(t) \\
& \quad=\left|\nu_{\alpha \mathrm{R}}\right|^{2} \\
& \quad=\mu^{2} B_{0}^{2}(1-\bar{\beta})^{2}\left[\cos ^{2} \theta \frac{\sin \left(\Omega_{-} t\right)}{\Omega_{-}}-\sin ^{2} \theta \frac{\sin \left(\Omega_{+} t\right)}{\Omega_{+}}\right]^{2} .
\end{aligned}
$$

It is interesting to compare $P_{\nu_{\beta \mathrm{L}} \rightarrow \nu_{\alpha \mathrm{R}}}$ in Eq. (3.20) with the transition probability of spin-flavor oscillations of Dirac neutrinos with large transition magnetic moment in a constant transverse magnetic field studied in Ref. [18]. For this purpose we should set $\omega=0$ and replace $(1-\bar{\beta}) \rightarrow 1$ in Eq. (3.20). In this case, $\Omega_{+}=\Omega_{-}=$ $\sqrt{\left(\mu B_{0}\right)^{2}+\left(\delta m^{2} / 4 p\right)^{2}}$. Thus we reproduce the transition probability found in Ref. [18].

Now we can compare $P_{\nu_{\beta \mathrm{L}} \rightarrow \nu_{\alpha \mathrm{R}}}$ in Eq. (3.20) with the corresponding transition probability for neutrino spinflavor oscillations in a plane electromagnetic wave found in Ref. [7]. Using Eq. (21) in Ref. [7] and omitting the neutrino matter interaction there, since here we study the neutrino interaction with an electromagnetic wave only, 
one obtains the following transition probability for $\nu_{\beta \mathrm{L}} \rightarrow \nu_{\alpha \mathrm{R}}$ oscillations:

$$
\begin{aligned}
& P_{\nu_{\beta \mathrm{L}} \rightarrow \nu_{\alpha \mathrm{R}}}(t) \\
& =\frac{\mu^{2} B_{0}^{2}(1-\bar{\beta})^{2}}{\mu^{2} B_{0}^{2}(1-\bar{\beta})^{2}+\left[\delta m^{2} A(\theta) / 4 p \pm \omega(1-\bar{\beta}) / 2\right]^{2}} \\
& \quad \times \sin ^{2}\left(\sqrt{\mu^{2} B_{0}^{2}(1-\bar{\beta})^{2}+\left[\delta m^{2} A(\theta) / 4 p \pm \omega(1-\bar{\beta}) / 2\right]^{2}} t\right) .
\end{aligned}
$$

If we consider $\nu_{e \mathrm{~L}} \rightarrow \nu_{\mu \mathrm{R}}$ transitions (see below), the function $A(\theta)$ reads $A(\theta)=(1+\cos 2 \theta) / 2$ [19]. The signs \pm in Eq. (3.21) correspond to different polarizations of the wave.

One can see that the result of Ref. [7], shown in Eq. (3.21), cannot be reproduced by Eq. (3.20), except in the trivial case $\theta=0$. However, the situation of a zero mixing angle between active neutrinos was recently excluded experimentally in Ref. [3], where it was shown that the remaining mixing angle $\theta_{13}$ is nonzero. Moreover, basing on Eq. (3.20), one cannot expect the appearance of the resonant amplification of spin-flavor oscillations, which can result from Eq. (3.21) and was claimed in Ref. [7].

The mentioned discrepancy is because of the unsubstantiated account of the neutrino vacuum oscillations phase $\delta m^{2} A(\theta) / 4 p$ in the dynamics of the neutrino spin in Ref. [7]. In Sec. II, using the method of the exact solution of the Dirac-Pauli equation, we have confirmed that the results of Ref. [7] are applicable for the description of the neutrino spin evolution within one mass eigenstate. Thus, in the present work, we have generalized the findings of Ref. [7] to correctly treat neutrino spin-flavor oscillations.

To analyze Eq. (3.20) we suppose that $\delta \Omega \ll \Omega_{ \pm}$, where $\delta \Omega=\left(\Omega_{+}-\Omega_{-}\right) / 2$. In this situation, the function $P_{\nu_{\beta \mathrm{L}} \rightarrow \nu_{\alpha \mathrm{R}}}$ becomes rapidly oscillating, being modulated by a slowly varying envelope function. Therefore we can apply the method of the analysis of this envelope function developed in Refs. [20,21]. Let us represent $P_{\nu_{\beta \mathrm{L}} \rightarrow \nu_{\alpha \mathrm{R}}}$ in the form

$$
\begin{aligned}
P_{\nu_{\beta \mathrm{L}} \rightarrow \nu_{\alpha \mathrm{R}}}(t) & =A_{\mathrm{eff}}(t) \sin ^{2}\left(\Omega_{0} t\right), \\
A_{\mathrm{eff}}(t) & =A_{\text {min }}+2 \delta A \sin ^{2}(\delta \Omega t),
\end{aligned}
$$

where

$$
\begin{aligned}
A_{\min } & =\frac{\left[\mu B_{0}(1-\bar{\beta}) \cos 2 \theta\right]^{2}}{\Omega_{0}^{2}}\left(1+\frac{2 \delta \Omega}{\cos 2 \theta \Omega_{0}}\right), \\
\delta A & =\frac{\left[\mu B_{0}(1-\bar{\beta}) \sin 2 \theta\right]^{2}}{2 \Omega_{0}^{2}}
\end{aligned}
$$

are the minimal value and the amplitude of the envelope function $A_{\text {eff }}(t)$. In Eqs. (3.22) and (3.23), $\Omega_{0}=$ $\sqrt{\mu^{2} B_{0}^{2}(1-\bar{\beta})^{2}+\left(\delta m^{2} / 4 p\right)^{2}}$.

The behavior of the transition probability for the $\nu_{e \mathrm{~L}} \rightarrow$ $\nu_{\mu \mathrm{R}}$ oscillations channel for different $\omega$ is shown in Fig. 2. One can see that $P_{\nu_{e \mathrm{~L}} \rightarrow \nu_{\mu \mathrm{R}}}$ is a rapidly oscillating function. Therefore, the averaged signal $\bar{P}(t)=A_{\min } / 2+\delta A \sin ^{2}(\delta \Omega t)$ is detected. The maximal averaged transition probability reads

$$
\bar{P}_{\max }=\frac{\left[\mu B_{0}(1-\bar{\beta})\right]^{2}}{2 \Omega_{0}^{2}}\left(1+2 \cos 2 \theta \frac{\delta \Omega}{\Omega_{0}}\right) .
$$

The envelope function $A_{\text {eff }}(t)$ and the averaged transition probability $\bar{P}(t)$ are also shown in Fig. 2.

The maximal averaged transition probability, calculated using Eq. (3.24) with the parameters corresponding to Figs. 2(a) and 2(b), is $\bar{P}_{\max } \approx 0.35$. This result is in agreement with Figs. 2(a) and 2(b). We can also estimate

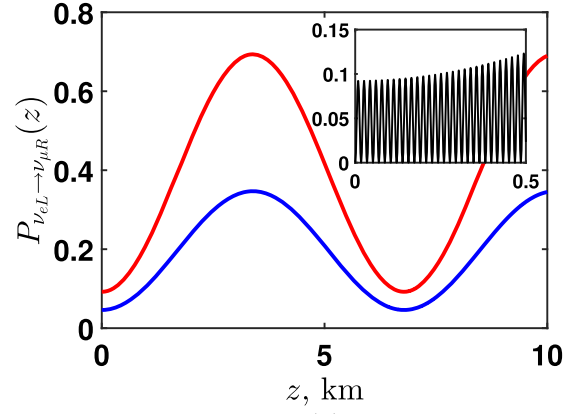

(a)

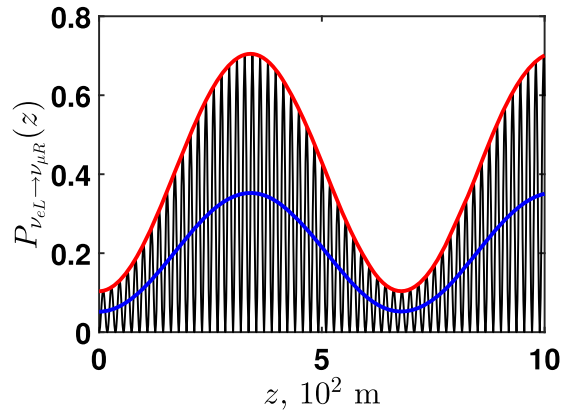

(b)

FIG. 2. The transition probability for the $\nu_{e \mathrm{~L}} \rightarrow \nu_{\mu \mathrm{R}}$ oscillations channel for different $\omega$ versus the propagation distance $z=t$, based on Eq. (3.22). The neutrino parameters are $p=1 \mathrm{keV}, m_{1} \approx m_{2}=1 \mathrm{eV}, \delta m^{2}=7.6 \times 10^{-5} \mathrm{eV}^{2}, \theta=0.6$ [17], and $\mu=10^{-11} \mu_{\mathrm{B}}$ [22], where $\mu_{\mathrm{B}}$ is the Bohr magneton. The amplitude of the electromagnetic wave $B_{0}=10^{18} \mathrm{G}$. (a) $\omega=10^{12} \mathrm{~s}^{-1}$; (b) $\omega=10^{13} \mathrm{~s}^{-1}$. The envelope function $A_{\text {eff }}(t)$ and the averaged transition probability $\bar{P}(t)$ are depicted by the red and blue lines, respectively. We do not show the actual behavior of the transition probability in panel (a) since it oscillates very rapidly. Instead we represent it in the inset in panel (a) at the small propagation distance $z<500 \mathrm{~m}$. 
the modulation length $L=\pi / \delta \Omega$. Using Eq. (3.22), one gets that

$$
\delta \Omega=\frac{\omega \delta m^{2}}{32 \Omega_{0} p^{3}}\left(m_{1}^{2}+m_{2}^{2}\right)
$$

Basing on the parameters corresponding to Fig. 2(a) and Eq. (3.25), we obtain that $L \approx 7.2 \mathrm{~km}$, whereas $L \approx 720 \mathrm{~m}$ for Fig. 2(b). These values of $L$ are in agreement with Fig. 2.

As an application of the obtained results, we consider the possibility of $\nu_{e \mathrm{~L}} \rightarrow \nu_{\mu \mathrm{R}}$ spin-flavor oscillations of astrophysical neutrinos in the vicinity of NS. A magnetic field with the strength $\sim 10^{18} \mathrm{G}$, chosen in Fig. 2, is slightly weaker than the strongest field allowed in NS [23]. The electromagnetic emission of pulsars ranges from radio to X-ray wavelengths [24]. The frequencies adopted in Fig. 2 correspond to wavelengths $\lambda=1.8 \mathrm{~mm}$ in Fig. 2(a) and $\lambda=0.18 \mathrm{~mm}$ in Fig. 2(b). These wavelengths lie in the infrared region. The electromagnetic emission of NS with such wavelengths was described in Ref. [25].

Note that further enhancement of the frequency is inexpedient since $\delta \Omega$ becomes comparable with $\Omega_{ \pm}$then. In principle, using our approach, we can also analyze spin-flavor oscillations in electromagnetic waves with wavelengths in the range: (several $\mathrm{mm})<\lambda<$ (several tens of $\mathrm{cm}$ ), emitted by radio pulsars. For a radio pulsar, $L$ is greater than that shown in Fig. 2.

An electromagnetic wave, which causes neutrino spinflavor oscillations, can be considered as plane only in the near field zone. In the far field zone, a wave should be taken as spherical, with its amplitude decreasing as $1 / z$. The size of the near field zone was estimated in Ref. [26] as $l \sim D^{2} / \lambda \sim D^{2} \omega$, where $D$ is the length scale of the region where a wave is emitted. In case of a pulsar, we can take that $D \sim 10^{4} \mathrm{~cm} \mathrm{[27].} \mathrm{Thus,} \mathrm{we} \mathrm{get} \mathrm{that} l \sim 3 \times 10^{4} \mathrm{~km}$ for the case shown in Fig. 2(a), and $l \sim 3 \times 10^{5} \mathrm{~km}$ for Fig. 2(b). The obtained values of $l$ are much greater than $L$ in Fig. 2. Hence the approximation of a plane electromagnetic wave is valid.

We studied neutrino spin-flavor oscillations in constant in space magnetic fields in Refs. [13,18]. Although neutrino oscillations in a constant field are not suppressed by the factor $(1-\bar{\beta}) \ll 1$, cf. Eq. (3.20), the analytical expressions for the transition probabilities, derived in Refs. $[13,18]$, cannot be applied for neutrinos propagating near NS at distances $R_{\mathrm{NS}}<z \lesssim l$, where $R_{\mathrm{NS}} \sim 10 \mathrm{~km}$ is the typical NS radius. Indeed, one cannot neglect the coordinate dependence of the magnetic field in NS at such distances; i.e., we have to take $B(z) \sim B_{\text {surf }}(R / z)^{3}$, where $B_{\text {surf }}$ is the magnetic field at the NS surface. Thus only a numerical transition probability can be obtained by solving the effective Schrödinger equation with $B=B(z)$. On the contrary, the analytical expression in Eq. (3.20) is valid for the distances $z$ up to $l$.
The most powerful neutrino emission takes place during a supernova explosion. In several minutes after an explosion, the neutrino flux becomes rather weak to be detected. Nevertheless, when the NS temperature is above $10^{7} \mathrm{~K} \sim 1 \mathrm{keV}$, neutrinos carry away energy from NS and are an effective tool for the NS cooling [28]. That is why we take the neutrino energy $\sim 1 \mathrm{keV}$ in Fig. 2. Thus such neutrinos, produced in modified Urca processes in the NS core, are subject to the described spin-flavor oscillations. Basing on $\bar{P}_{\max }$ in Fig. 2, one gets that about $35 \%$ of $\nu_{e \mathrm{~L}}$ is converted to $\nu_{\mu \mathrm{R}}$.

It should be noted that the chosen parameters $B_{0}$ and $p$ correspond to $\delta A \lesssim 1$ in Eq. (3.23). It guarantees that the transition probability is not suppressed significantly. We mention that the study of the electromagnetic emission of pulsars is a rapidly developing area of modern astrophysics. Despite the recent advances in the description of the pulsars radiation [29], there is no self-consistent model of this process [30]. In a realistic situation, the amplitude of an electromagnetic wave is likely to be smaller that $10^{18} \mathrm{G}$. In the case, when $\mu B_{0}(1-\bar{\beta}) \ll \delta m^{2} / 4 p$, using Eq. (3.24), the maximal averaged transition probability is

$$
\bar{P}_{\max } \approx 2\left[\mu B_{0}(1-\bar{\beta}) \frac{2 p}{\delta m^{2}}\right]^{2}<0.35
$$

Thus, the value $\bar{P}_{\max }=0.35$, obtained above, is the upper bound on the maximal transition probability in NS since we supposed that $B_{0}$ equals to the strongest magnetic field, which can exist in such a system.

At the end of this section, we discuss the general issue on the approximations used in the derivation of the main results. To obtain Eq. (3.10) we supposed that the neutrino mass eigenstates are localized on their classical trajectories. However, to derive Eq. (3.19) one has to assume that the neutrino wave packets are wide. The latter assumption is necessary for a significant overlap of different mass eigenstates for the oscillations process to occur. Formally we should require that $\ell \gg \delta \Omega^{-1}$, where $\ell$ is the width of the wave packet. However we can suggest that pointlike neutrinos with equal velocities are coherently emitted during the time interval $\Delta t \gg \delta \Omega^{-1}$. In this case, one deals with an effectively wide neutrino wave packet consisting of mass eigenstates propagating with a certain velocity. Thus we can reconcile both assumptions necessary to get Eqs. (3.10) and (3.19).

\section{SPIN-FLAVOR OSCILLATIONS: INFLUENCE OF DIAGONAL MAGNETIC MOMENTS}

In this section, we discuss the influence of the diagonal magnetic moments $\mu_{a}$ on the neutrino spin-flavor oscillations in a plane electromagnetic wave. In our analysis, we still suppose that $\mu_{a}$ is small: $\mu_{a} \ll \mu$. 
If one accounts for $\mu_{a}$, the main formalism, developed in Sec. III, remains practically unchanged. First, Eq. (3.5) is modified, so that the two component spinor $v_{s}$, entering the bispinor $u_{a s}$, becomes dependent on time and $\mu_{a}$, i.e., $v_{s} \rightarrow v_{a s}(t)$. The temporal dependence of $v_{a s}$ is given by Eq. (2.4). We can choose two independent spin states $v_{0 s}$ in Eq. (2.4) as $v_{0+}^{\mathrm{T}}=(1,0)$ and $v_{0-}^{\mathrm{T}}=(0,1)$. In this situation, both $v_{a \pm}(t)$ and $u_{a \pm}$, corresponding to the opposite spin states, are orthogonal.

Second, the matrix elements in Eq. (3.8) become quite cumbersome. The calculation of the matrix elements is provided in Appendix in the general form. Using Eq. (A4) and making the calculations similar to those in Sec. III, we derive the analogue of Eq. (3.11),

$$
\begin{aligned}
& \mathrm{i} \frac{\mathrm{d}}{\mathrm{d} t} \Psi=H_{\text {eff }} \Psi, \\
& H_{\text {eff }}=-\mu B_{0}(1-\bar{\beta})\left(\begin{array}{cccc}
0 & 0 & \epsilon_{+} & \exp \left(\mathrm{i} \Phi_{-} t\right) \\
0 & 0 & \exp \left(\mathrm{i} \Phi_{+} t\right) & \epsilon_{-} \\
\epsilon_{+}^{*} & \exp \left(-\mathrm{i} \Phi_{+} t\right) & 0 & 0 \\
\exp \left(-\mathrm{i} \Phi_{-} t\right) & \epsilon_{-}^{*} & 0 & 0
\end{array}\right) \text {, }
\end{aligned}
$$

where

$$
\epsilon_{ \pm}=\frac{B_{0}}{\omega}\left[ \pm\left(\mu_{1}+\mu_{2}\right) \mp \mu_{1} e^{ \pm \mathrm{i} \omega t(1-\bar{\beta})} \mp \mu_{2} e^{\mp \mathrm{i} \omega t(1-\bar{\beta})}\right] .
$$

In Eqs. (4.1) and (4.2), we set $z=\bar{\beta} t$ and assume that $m_{1} \approx m_{2}$ in the off-diagonal terms $\sim \exp \left( \pm \mathrm{i} \Phi_{ \pm} t\right)$ in $H_{\text {eff }}$.

Since we discuss the situation when $m_{1} \approx m_{2}$, it is reasonable to take that $\mu_{1} \approx \mu_{2}=\mu^{\prime}$. In this case, $\epsilon_{ \pm}= \pm \epsilon$, where $\epsilon=4 \mu^{\prime} B_{0} \sin ^{2}[\omega t(1-\bar{\beta}) / 2] / \omega$. Then, we can derive the analogue of Eq. (3.15),

$$
\begin{aligned}
\mathrm{i} \frac{\mathrm{d}}{\mathrm{d} t} \tilde{\Psi} & =\tilde{H}_{\text {eff }} \tilde{\Psi}, \\
\tilde{H}_{\text {eff }} & =\left(\begin{array}{cccc}
\Phi_{-} / 2 & 0 & -\mu B_{0}(1-\bar{\beta}) \epsilon & -\mu B_{0}(1-\bar{\beta}) \\
0 & \Phi_{+} / 2 & -\mu B_{0}(1-\bar{\beta}) & \mu B_{0}(1-\bar{\beta}) \epsilon \\
-\mu B_{0}(1-\bar{\beta}) \epsilon & -\mu B_{0}(1-\bar{\beta}) & -\Phi_{+} / 2 & 0 \\
-\mu B_{0}(1-\bar{\beta}) & \mu B_{0}(1-\bar{\beta}) \epsilon & 0 & -\Phi_{-} / 2
\end{array}\right) .
\end{aligned}
$$

Should one have the solution of Eq. (4.3), the transition probability for $\nu_{\beta \mathrm{L}} \rightarrow \nu_{\alpha \mathrm{R}}$ oscillations reads

$$
P_{\nu_{\beta \mathrm{L}} \rightarrow \nu_{\alpha \mathrm{R}}}(t)=\left|\cos \theta \tilde{a}_{1+}(t)-\sin \theta \tilde{a}_{2+}(t)\right|^{2} .
$$

One can show that Eq. (4.4) is equivalent to $P_{\nu_{\beta \mathrm{L}} \rightarrow \nu_{\alpha \mathrm{R}}}$ calculated using $a_{a+}(t)$, i.e., based on the solution of Eq. (4.1), as $\nu_{\alpha \mathrm{R}}(z, t)$ in Eq. (3.19).

For the illustration of the influence of the diagonal magnetic moments on neutrino spin-flavor oscillations in a plane electromagnetic wave, we consider $\nu_{e \mathrm{~L}} \rightarrow \nu_{\mu \mathrm{R}}$ with the same parameters as in Sec. III. In Figs. 3 and 4, we show $P_{\nu_{e \mathrm{~L}} \rightarrow \nu_{\mu \mathrm{R}}}$ based on the numerical solution of Eq. (4.3) for different $\mu^{\prime}$ (or $\epsilon_{0}=\mu^{\prime} B_{0} / \omega$ ) and $\omega$. The upper and lower envelope functions, as well as the averaged transition probability, are also depicted in Figs. 3 and 4. To build these envelope functions we use the spline interpolation of the maxima and minima of $P_{\nu_{e \mathrm{~L}} \rightarrow \nu_{\mu \mathrm{R}}}$, respectively.
Note that, as in Fig. 2(a), we do not represent the evolution of $P_{\nu_{e \mathrm{~L}} \rightarrow \nu_{\mu \mathrm{R}}}(z)$ in Fig. 3 since this function is rapidly oscillating and, hence, is indistinguishable. Instead, we just show the envelope functions and the averaged transition probability in Fig. 3.

One can see that $P_{\nu_{e \mathrm{~L}} \rightarrow \nu_{\mu \mathrm{R}}}$ and the envelope functions in Figs. 3(a) and 4(a), corresponding to $\mu^{\prime}=0$, coincide with the curves shown in Figs. 2(a) and 2(b), which were built on the basis of the exact solution of Eq. (3.15); cf. Eqs. (3.16), (3.17), and (3.20). It is also interesting to note that $P_{\nu_{e \mathrm{~L}} \rightarrow \nu_{\mu \mathrm{R}}}$ and the envelope function for spin-flavor oscillations of neutrinos with $\mu^{\prime} \sim 10^{-2} \mu \ll \mu$, shown in Figs. 3(d) and 4(d), significantly differ from the case $\mu^{\prime}=0$ shown in Figs. 3(a) and 4(a).

Basing on Figs. 3(b)-3(d) and 4(b)-4(d), one can conclude that the averaged transition probability diminishes if one accounts for $\mu^{\prime}>0$ in the system. Thus the situation when $\mu_{a}=0$, studied in Sec. III, is more preferable from the point of view of phenomenological applications. 


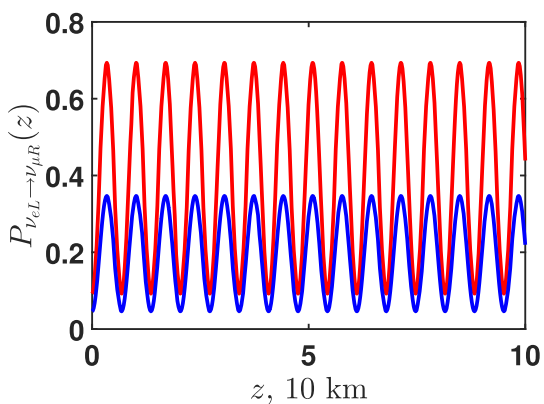

(a)

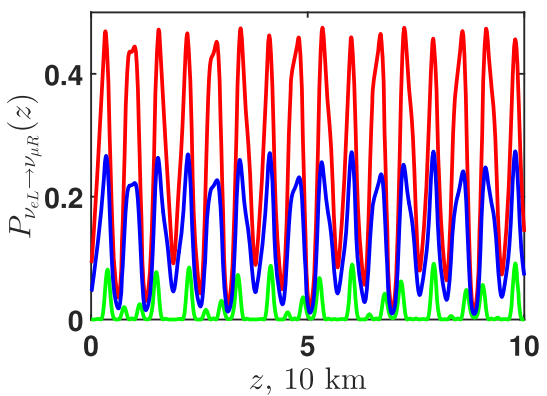

(c)

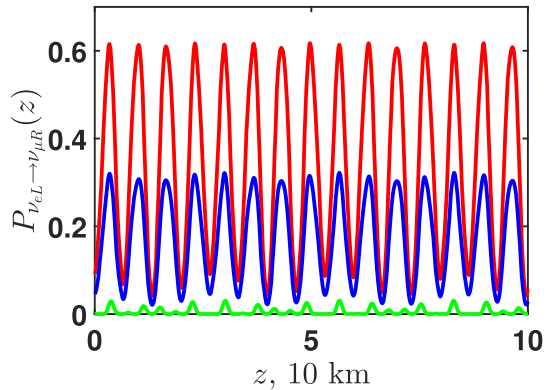

(b)

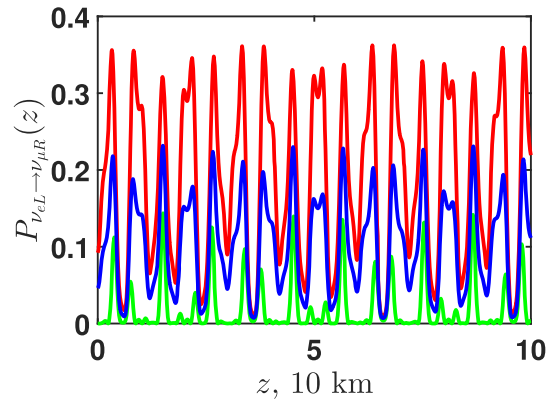

(d)

FIG. 3. The upper (red lines) and lower (green lines) envelope functions, as well as the averaged transition probability (blue lines), corresponding to Eq. (4.4), for $\nu_{e \mathrm{~L}} \rightarrow \nu_{\mu \mathrm{R}}$ oscillations in the electromagnetic wave versus the distance $z$ passed by the neutrino beam for different diagonal magnetic moment $\mu^{\prime}$, based on the numerical solution of Eq. (4.3). The parameters of the neutrino system and $B_{0}$ are the same as in Fig. 2. The frequency of the electromagnetic wave is $\omega=10^{12} \mathrm{~s}^{-1}$. (a) $\mu^{\prime}=0\left(\epsilon_{0}=0\right)$; (b) $\mu^{\prime}=2.8 \times 10^{-3} \mu=2.8 \times 10^{-14} \mu_{\mathrm{B}}$ $\left(\epsilon_{0}=0.1\right)$; (c) $\mu^{\prime}=5.5 \times 10^{-3} \mu=5.5 \times 10^{-14} \mu_{\mathrm{B}}\left(\epsilon_{0}=0.2\right)$; (d) $\mu^{\prime}=8.3 \times 10^{-3} \mu=8.3 \times 10^{-14} \mu_{\mathrm{B}}\left(\epsilon_{0}=0.3\right)$.

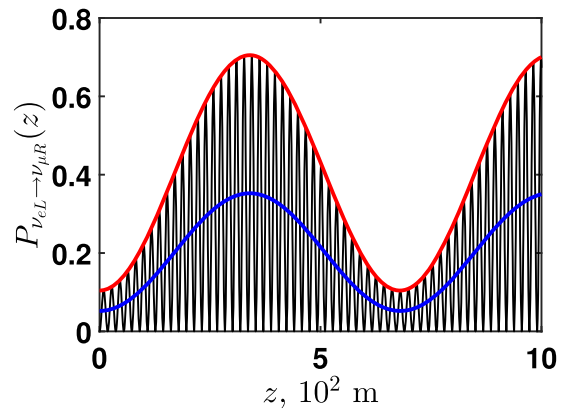

(a)

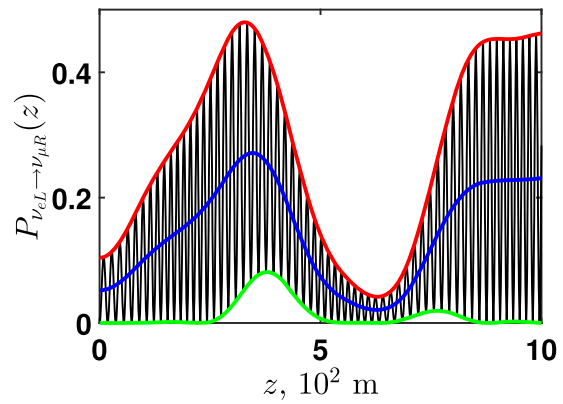

(c)

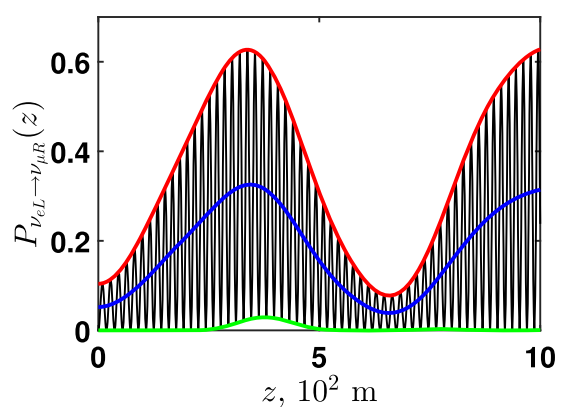

(b)

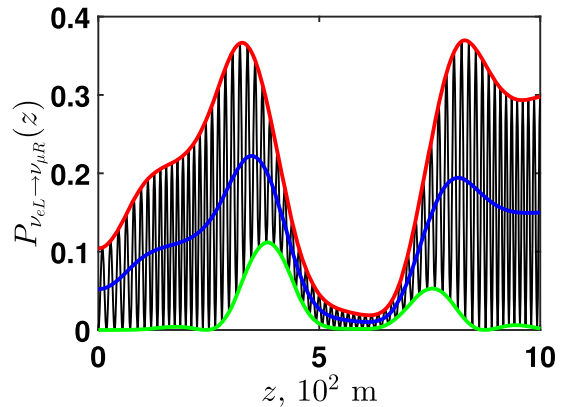

(d)

FIG. 4. The transition probability (black lines) for $\nu_{e \mathrm{~L}} \rightarrow \nu_{\mu \mathrm{R}}$ oscillations, as well as the upper (red lines) and lower (green lines) envelope functions, and the averaged transition probability (blue lines) for this oscillations channel, based on the numerical solution of Eq. (4.3). The parameters of the neutrino system and the electromagnetic wave are the same as in Fig. 3, except for the frequency, which is now equal to $\omega=10^{13} \mathrm{~s}^{-1}$. 


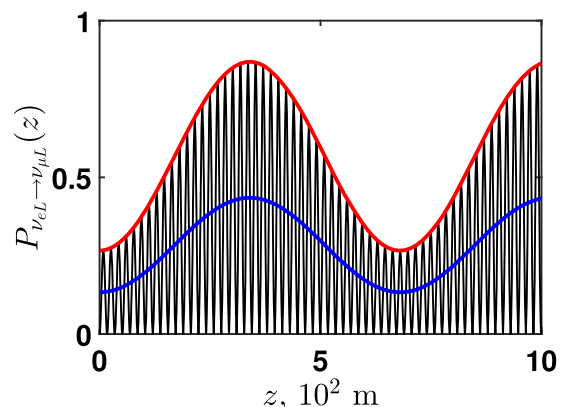

(a)

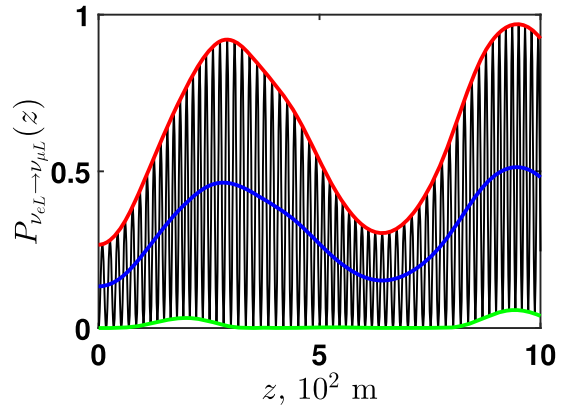

(c)

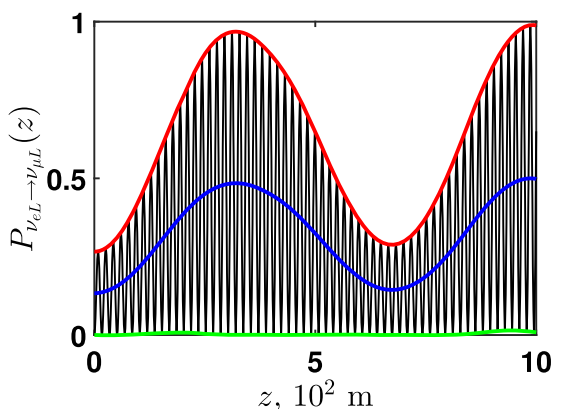

(b)

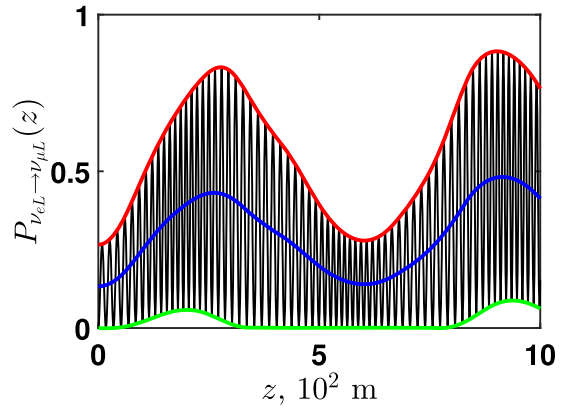

(d)

FIG. 5. The transition probability (black lines) for $\nu_{e \mathrm{~L}} \rightarrow \nu_{\mu \mathrm{L}}$ oscillations, as well as the upper (red lines) and lower (green lines) envelope functions, and the averaged transition probability (blue lines) for this oscillations channel, based on the numerical solution of Eq. (4.3). The parameters of the neutrino system and the electromagnetic wave are the same as in Fig. 4.

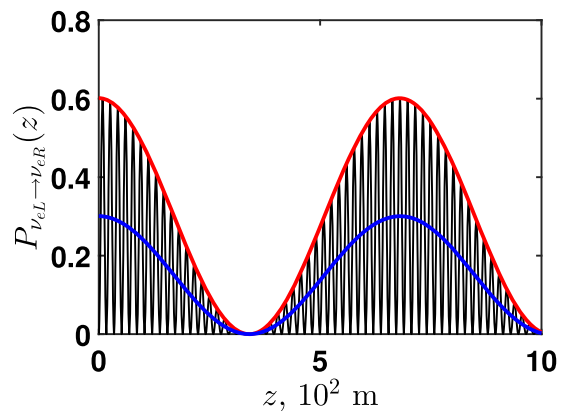

(a)

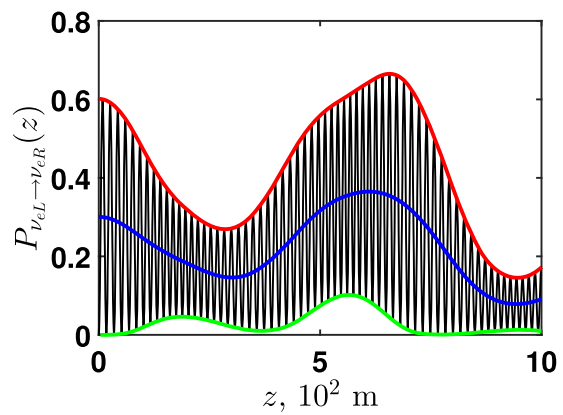

(c)

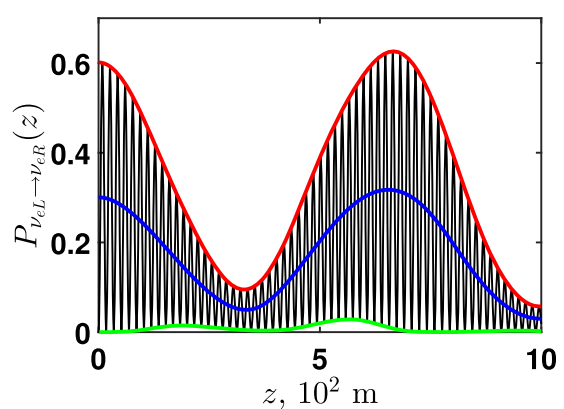

(b)

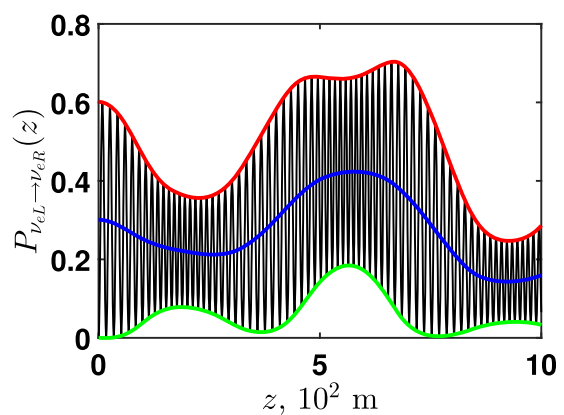

(d)

FIG. 6. The transition probability (black lines) for $\nu_{e \mathrm{~L}} \rightarrow \nu_{e \mathrm{R}}$ oscillations, as well as the upper (red lines) and lower (green lines) envelope functions, and the averaged transition probability (blue lines) for this oscillations channel, based on the numerical solution of Eq. (4.3). The parameters of the neutrino system and the electromagnetic wave are the same as in Fig. 4. 


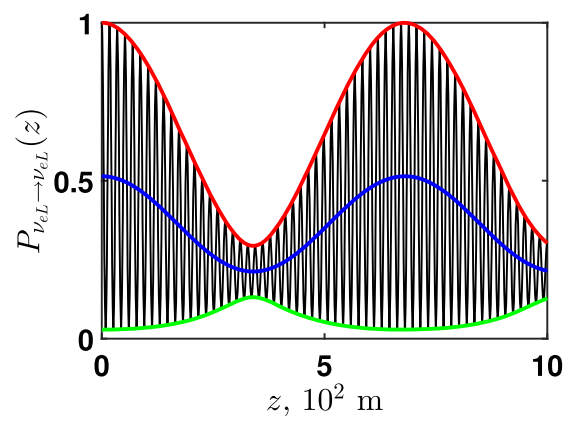

(a)

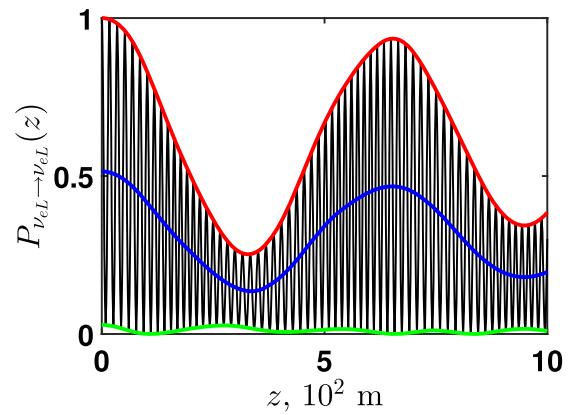

(c)

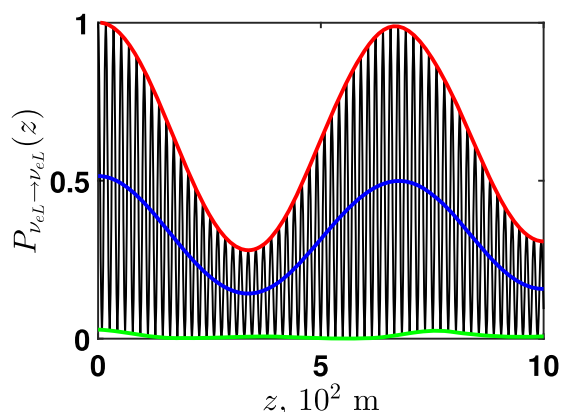

(b)

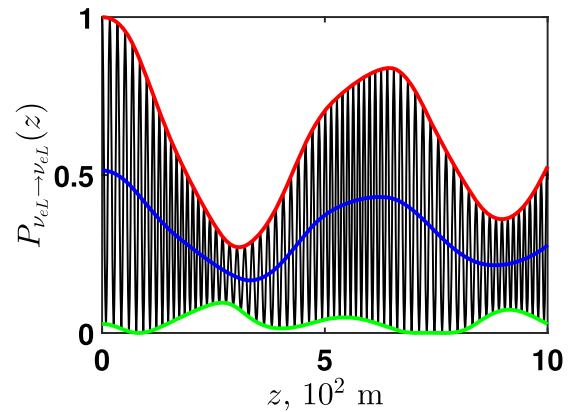

(d)

FIG. 7. The survival probability (black lines) for $\nu_{e \mathrm{~L}} \rightarrow \nu_{e \mathrm{~L}}$ oscillations, as well as the upper (red lines) and lower (green lines) envelope functions, and the averaged survival probability (blue lines) for this oscillations channel, based on the numerical solution of Eq. (4.3). The parameters of the neutrino system and the electromagnetic wave are the same as in Fig. 4.

Besides $\nu_{e \mathrm{~L}} \rightarrow \nu_{\mu \mathrm{R}}$ transitions, we can study other channels of neutrino oscillations on the basis of the numerical solution of Eq. (4.3). For example, in Fig. 5, we demonstrate the transition probability, the upper and lower envelope functions, and the averaged transition probability for $\nu_{e \mathrm{~L}} \rightarrow \nu_{\mu \mathrm{L}}$ oscillations. These dependencies for $\nu_{e \mathrm{~L}} \rightarrow \nu_{e \mathrm{R}}$ oscillations are shown in Fig. 6. The survival probability for oscillations $\nu_{e \mathrm{~L}} \rightarrow \nu_{e \mathrm{~L}}$, the upper and lower envelope functions, and the averaged survival probability are given in Fig. 7.

One can see in Figs. 4-6 that $P_{\nu_{\mathrm{e}} \rightarrow \nu_{\mu \mathrm{R}}}=P_{\nu_{e \mathrm{~L}} \rightarrow \nu_{\mu \mathrm{L}}}=$ $P_{\nu_{e \mathrm{~L}} \rightarrow \nu_{e \mathrm{R}}}=0$ at $z=0$, whereas $P_{\nu_{e \mathrm{~L}} \rightarrow \nu_{e \mathrm{~L}}}(z=0) \stackrel{\nu_{e \mathrm{~L}} \rightarrow \nu_{\mu \mathrm{L}}}{=}$ in Fig. 7; i.e., the initial condition is fulfilled. Figures 4-7 are built for $\omega=10^{13} \mathrm{~s}^{-1}$. The analogues of these dependencies for $\omega=10^{12} \mathrm{~s}^{-1}$ are not presented here since the transition and survival probabilities are rapidly oscillating functions and, hence, are quite difficult for the analysis.

We also mention that, in this section, we study only the situation when $\omega \geq 10^{12} \mathrm{~s}^{-1}$. The numerical solution of Eq. (4.3) for $\omega<10^{12} \mathrm{~s}^{-1}$ (i.e., the situation corresponding to electromagnetic waves emitted by a radio pulsar; cf. Sec. III) is quite problematic since Eq. (4.3) becomes extremely stiff then.

\section{SPIN-FLAVOR OSCILLATIONS: GREAT DIAGONAL MAGNETIC MOMENTS}

In this section, we study spin-flavor oscillations of neutrinos with great diagonal magnetic moments in a plane electromagnetic wave.
In some extensions of the standard model, e.g., in a minimally extended standard model, diagonal magnetic moments of neutrino are proportional to neutrino masses [31]. The transition magnetic moment is much smaller than diagonal ones because of the Glashaw-Iliopoulos-Maiani (GIM) mechanism [ [6], pp. 461-479]. Considering the situation of great diagonal magnetic moments, we can derive analytically the transition probability of neutrino spin-flavor oscillations in an electromagnetic wave for such neutrinos.

As in Sec. III, we study the evolution of neutrino mass eigenstates. The Dirac equation for $\psi_{a}$ coincides with that in Eqs. (3.3) and (3.4). However we should take that $V=0$ since we study the situation when $\mu_{a} \gg \mu$.

Since the transition magnetic moment is not taken into account, one has the following qualitative evolution of the neutrino spin and mass eigenstates. There is independent spin precession within each mass eigenstate owing to the interaction of the neutrino magnetic moment $\mu_{a}$ with the electromagnetic field of the wave. The transition between different mass eigenstates is solely due to the presence of the nonzero vacuum mixing in Eq. (3.2).

Therefore, to find the transition probability for $\nu_{\beta \mathrm{L}} \rightarrow$ $\nu_{\alpha \mathrm{R}}$ oscillations, we can use Eqs. (2.2)-(2.4), as well as the results of Ref. [18], where spin-flavor oscillations of neutrinos with arbitrary magnetic moments were studied in the constant magnetic field. We assume that neutrinos are ultrarelativistic particles and adopt the quasiclassical approximation from the very beginning. Omitting some 
intermediate calculations, we just give the final result for the transition probability,

$$
P_{\nu_{\beta \mathrm{L}} \rightarrow \nu_{\alpha \mathrm{R}}}(t)=\sin ^{2}(2 \theta)\left[\frac{1}{4}\left(A_{1}-A_{2}\right)^{2}+A_{1} A_{2} \sin ^{2}\left(\Phi_{\mathrm{vac}} t\right)\right],
$$

where

$$
A_{1,2}(t)=\frac{\mu_{1,2} B_{0}}{\sqrt{\mu_{1,2}^{2} B_{0}^{2}+\omega^{2} / 4}} \sin \left[\sqrt{\mu_{1,2}^{2} B_{0}^{2}+\omega^{2} / 4}\left(1-\beta_{1,2}\right) t\right]
$$

are the amplitudes of probabilities of spin oscillations within each mass eigenstate and $\Phi_{\text {vac }}=\delta m^{2} / 4 p$ is the phase of flavor oscillations in vacuum.

If we set $\omega=0$ and replace $\left(1-\beta_{1,2}\right) \rightarrow 1$ in Eq. (5.2), we get that $A_{1,2}=\sin \left(\mu_{1,2} B_{0} t\right)$. Thus Eq. (5.1) reproduces the transition probability for $\nu_{\beta \mathrm{L}} \rightarrow \nu_{\alpha \mathrm{R}}$ oscillations in a constant transverse magnetic field derived in Ref. [18] for the case $\mu_{a} \gg \mu$.

Although the transition probability obtained in Eqs. (5.1) and (5.2) is analytical, the situation when $\mu_{a} \gg \mu$ has very limited applicability from the point of view of phenomenology. The fact is that $\mu_{a} \sim 10^{-19} \mu_{\mathrm{B}}$ in the minimally extended standard model for reasonable values of neutrino masses [16]. Thus $P_{\nu_{\beta \mathrm{L}} \rightarrow \nu_{\alpha \mathrm{R}}} \ll 1$ for any $t$. That is why we do not provide the correction to $P_{\nu_{\beta \mathrm{L}} \rightarrow \nu_{\alpha \mathrm{R}}} \ll 1$ owing to $\mu \neq 0$ since such a quantity is really negligible.

\section{CONCLUSION}

In conclusion, we mention that, in the present work, we have studied spin and spin-flavor oscillations of Dirac neutrinos in a plane electromagnetic wave with the circular polarization. Our analysis was based on solving the initial condition problem for the system of flavor neutrinos interacting with external fields. This method was reviewed in Ref. [13]. The neutrino interaction with the electromagnetic wave is owing to the presence of the nonzero neutrino magnetic moments. This interaction was accounted for by using the exact solution of the Dirac-Pauli equation for massive fermions found in Ref. [11].

In Sec. II, we have considered neutrino spin oscillations in a plane electromagnetic wave within one neutrino mass eigenstate. We have obtained the exact expression for the transition probability for $\nu_{\mathrm{L}} \rightarrow \nu_{\mathrm{R}}$ oscillations in Eq. (2.5). This transition probability turns out to depend on both time and the spatial coordinate. However, for an ultrarelativistic neutrino, which is localized along its trajectory, the spatial coordinate dependence is transformed to the dependence on time only. In this case, we have reproduced the transition probability obtained in Ref. [7], where the quasiclassical approach for the description of the neutrino spin evolution in electromagnetic fields was developed.

Then, in Secs. III-V, we have generalized the approach in Sec. II to treat neutrino spin-flavor oscillations. In Sec. III, we have started with the analysis of the situation when only a great transition magnetic moment is present in the mass eigenstates basis. In this situation, one can find the analytic expression for the transition probability; cf. Eq. (3.20). Comparing the transition probabilities in Fig. 2 with the results of Ref. [20], one can see that spinflavor oscillations of Dirac neutrinos in an electromagnetic wave are analogous to oscillations in a twisting magnetic field.

The expression for the transition probability in Eq. (3.20) turns out to differ from the analogous result obtained in Ref. [7]; cf. Eq. (3.21). This discrepancy is due to the incorrect treatment of transitions between different neutrino flavor eigenstates in Ref. [7]. As we have mentioned above, the neutrino spin evolution within one neutrino mass eigenstate was accounted for correctly in Ref. [7]. Thus, in the present work, we have provided the valid generalization of the results of Ref. [7] for the description of neutrino spin-flavor oscillations in an electromagnetic wave.

Then, in Sec. III, we have discussed a possibility of an astrophysical application of the obtained results. We have studied $\nu_{e \mathrm{~L}} \rightarrow \nu_{\mu \mathrm{R}}$ oscillations in an electromagnetic wave emitted by a highly magnetized NS. We found that the upper bound on the averaged transition probability can reach 35\%; cf. Fig. 2. If the amplitude of an electromagnetic wave is less than $10^{18} \mathrm{G}$, which is likely to be the case in realistic astrophysical media, the maximal transition probability can be found in Eq. (3.26).

In Sec. IV, we have briefly discussed the influence of the diagonal magnetic moments on spin-flavor oscillations in an electromagnetic wave. We have assumed that diagonal magnetic moments, $\mu^{\prime} \sim 10^{-14} \mu_{\mathrm{B}}$, are much smaller than the transition one, $\mu \sim 10^{-11} \mu_{\mathrm{B}}$. This value of $\mu^{\prime}$ is in agreement with the upper bound on diagonal magnetic moments of Dirac neutrinos revealed in Ref. [32]. One can see in Figs. 3 and 4 that, if diagonal magnetic moments are taken into account, the averaged transition probability is less than in the case when only a transition magnetic moment is accounted for. Besides the $\nu_{e \mathrm{~L}} \rightarrow \nu_{\mu \mathrm{R}}$ oscillations channel, we have studied other types of neutrino oscillations like $\nu_{e \mathrm{~L}} \rightarrow \nu_{\mu \mathrm{L}}, \nu_{e \mathrm{~L}} \rightarrow \nu_{e \mathrm{R}}$, and $\nu_{e \mathrm{~L}} \rightarrow \nu_{e \mathrm{~L}}$ in Sec. IV; cf. Figs. 5-7.

Finally, in Sec. V, we have provided the analytical transition probability for neutrino spin-flavor oscillations in an electromagnetic wave in cases when diagonal magnetic moments dominate over the transition one; cf. Eqs. (5.1) and (5.2). This situation can take place, e.g., in the minimally extended standard model, where 
the transition magnetic moment is suppressed by the GIM mechanism [ [6], pp. 461-479]. However, this result is of limited phenomenological applicability since the diagonal magnetic moments are extremely small in this model.

\section{ACKNOWLEDGMENTS}

This work was partially supported by the Competitiveness Improvement Program at the Tomsk State University, RFBR (Grant No. 18-02-00149a), and the Foundation for the Advancement of Theoretical Physics and Mathematics "BASIS." I am also thankful to $\mathrm{S}$. Dvornikov for the assistance in numerical simulations.

\section{APPENDIX: MATRIX ELEMENTS OF SPIN INTERACTION}

In this Appendix, we compute the matrix elements of the neutrino spin interaction with a plane circularly polarized electromagnetic wave between two component spinors corresponding to different mass eigenstates.

First, we replace $\mu \rightarrow \mu_{a}$ in Eq. (2.4). Then, we define $v_{a s}(t)$ corresponding to $v_{0 s}$, where $v_{0+}^{\mathrm{T}}=(1,0)$ and $v_{0-}^{\mathrm{T}}=(0,1)$. Basing on the unitarity of the evolution of the spinors in Eq. (2.4), one gets that $v_{a s}^{\dagger}(t) v_{a s^{\prime}}(t)=v_{0 s}^{\dagger} v_{0 s^{\prime}}=\delta_{s s^{\prime}}$; i.e., the spinors $v_{a s}(t)$ are orthogonal.

After a lengthy but straightforward calculation we obtain the matrix elements $v_{a s}^{\dagger}(t)(\boldsymbol{\sigma B}) v_{b s^{\prime}}(t), a \neq b$, which enter Eq. (3.6), in the following form:

$$
\begin{aligned}
& v_{1 \pm}^{\dagger}(\boldsymbol{\sigma} \mathbf{B}) v_{2 \pm}=\frac{B_{0}}{\sqrt{\mu_{1}^{2} B_{0}^{2}+\omega^{2} / 4} \sqrt{\mu_{2}^{2} B_{0}^{2}+\omega^{2} / 4}} \\
& \times\left\{ \pm\left(\mu_{1}+\mu_{2}\right) B_{0} \frac{\omega}{2} \sin \left[\sqrt{\mu_{1}^{2} B_{0}^{2}+\omega^{2} / 4} \phi\right] \sin \left[\sqrt{\mu_{2}^{2} B_{0}^{2}+\omega^{2} / 4} \phi\right]\right. \\
& +\mathrm{i} \mu_{2} B_{0} \sqrt{\mu_{1}^{2} B_{0}^{2}+\omega^{2} / 4} \cos \left[\sqrt{\mu_{1}^{2} B_{0}^{2}+\omega^{2} / 4} \phi\right] \sin \left[\sqrt{\mu_{2}^{2} B_{0}^{2}+\omega^{2} / 4} \phi\right] \\
& \left.-\mathrm{i} \mu_{1} B_{0} \sqrt{\mu_{2}^{2} B^{2}+\omega^{2} / 4} \sin \left[\sqrt{\mu_{1}^{2} B_{0}^{2}+\omega^{2} / 4} \phi\right] \cos \left[\sqrt{\mu_{2}^{2} B_{0}^{2}+\omega^{2} / 4} \phi\right]\right\}, \\
& v_{1-}^{\dagger}(\boldsymbol{\sigma} \mathbf{B}) v_{2+}=\frac{B_{0}}{\sqrt{\mu_{1}^{2} B_{0}^{2}+\omega^{2} / 4} \sqrt{\mu_{2}^{2} B_{0}^{2}+\omega^{2} / 4}} \\
& \times\left\{\left(\mu_{1} \mu_{2} B_{0}^{2}-\frac{\omega^{2}}{4}\right) \sin \left[\sqrt{\mu_{1}^{2} B_{0}^{2}+\omega^{2} / 4} \phi\right] \sin \left[\sqrt{\mu_{2}^{2} B_{0}^{2}+\omega^{2} / 4} \phi\right]\right. \\
& +\sqrt{\mu_{1}^{2} B_{0}^{2}+\omega^{2} / 4} \sqrt{\mu_{2}^{2} B_{0}^{2}+\omega^{2} / 4} \\
& \times \cos \left[\sqrt{\mu_{1}^{2} B_{0}^{2}+\omega^{2} / 4} \phi\right] \cos \left[\sqrt{\mu_{2}^{2} B_{0}^{2}+\omega^{2} / 4} \phi\right] \\
& +\mathrm{i} \frac{\omega}{2} \sqrt{\mu_{1}^{2} B_{0}^{2}+\omega^{2} / 4} \cos \left[\sqrt{\mu_{1}^{2} B_{0}^{2}+\omega^{2} / 4} \phi\right] \sin \left[\sqrt{\mu_{2}^{2} B_{0}^{2}+\omega^{2} / 4} \phi\right] \\
& \left.+\mathrm{i} \frac{\omega}{2} \sqrt{\mu_{2}^{2} B_{0}^{2}+\omega^{2} / 4} \sin \left[\sqrt{\mu_{1}^{2} B_{0}^{2}+\omega^{2} / 4} \phi\right] \sin \left[\sqrt{\mu_{2}^{2} B_{0}^{2}+\omega^{2} / 4} \phi\right]\right\}, \\
& v_{1+}^{\dagger}(\boldsymbol{\sigma} \mathbf{B}) v_{2-}=\frac{B_{0}}{\sqrt{\mu_{1}^{2} B_{0}^{2}+\omega^{2} / 4} \sqrt{\mu_{2}^{2} B_{0}^{2}+\omega^{2} / 4}} \\
& \times\left\{\left(\mu_{1} \mu_{2} B_{0}^{2}-\frac{\omega^{2}}{4}\right) \sin \left[\sqrt{\mu_{1}^{2} B_{0}^{2}+\omega^{2} / 4} \phi\right] \sin \left[\sqrt{\mu_{2}^{2} B_{0}^{2}+\omega^{2} / 4} \phi\right]\right. \\
& +\sqrt{\mu_{1}^{2} B_{0}^{2}+\omega^{2} / 4} \sqrt{\mu_{2}^{2} B_{0}^{2}+\omega^{2} / 4} \\
& \times \cos \left[\sqrt{\mu_{1}^{2} B_{0}^{2}+\omega^{2} / 4} \phi\right] \cos \left[\sqrt{\mu_{2}^{2} B_{0}^{2}+\omega^{2} / 4} \phi\right] \\
& -\mathrm{i} \frac{\omega}{2} \sqrt{\mu_{1}^{2} B_{0}^{2}+\omega^{2} / 4} \cos \left[\sqrt{\mu_{1}^{2} B_{0}^{2}+\omega^{2} / 4} \phi\right] \sin \left[\sqrt{\mu_{2}^{2} B_{0}^{2}+\omega^{2} / 4} \phi\right] \\
& \left.-\mathrm{i} \frac{\omega}{2} \sqrt{\mu_{2}^{2} B_{0}^{2}+\omega^{2} / 4} \sin \left[\sqrt{\mu_{1}^{2} B_{0}^{2}+\omega^{2} / 4} \phi\right] \sin \left[\sqrt{\mu_{2}^{2} B_{0}^{2}+\omega^{2} / 4} \phi\right]\right\},
\end{aligned}
$$

where $\phi=t-z$. 
In Sec. IV, we are interested in the situation of small diagonal magnetic moments: $\mu_{a} \ll \mu$. Expanding Eqs. (A1)-(A3) in $\mu_{a} B_{0}$ we get that only $v_{1 \pm}^{\dagger}(\boldsymbol{\sigma} \mathbf{B}) v_{2 \pm}$ contains the contribution linear in $\mu_{a}$,

$$
v_{1 \pm}^{\dagger}(\boldsymbol{\sigma} \mathbf{B}) v_{2 \pm}=\frac{B_{0}^{2}}{\omega}\left[ \pm\left(\mu_{1}+\mu_{2}\right) \mp \mu_{1} e^{ \pm i \omega \phi} \mp \mu_{2} e^{\mp i \omega \phi}\right] .
$$

The expressions for $v_{1 \pm}^{\dagger}(\boldsymbol{\sigma} \mathbf{B}) v_{2 \mp}$ in Eqs. (A2) and (A3) are $\sim \mu_{a}^{2}$. Hence we should use the zero order approximation in Eq. (3.8) for them.

[1] Y. Fukuda et al. (Super-Kamiokande Collaboration), Evidence for Oscillation of Atmospheric Neutrinos, Phys. Rev. Lett. 81, 1562 (1998).

[2] Q. R. Ahmad et al. (SNO Collaboration), Direct Evidence for Neutrino Flavor Transformation from Neutral Current Interactions in the Sudbury Neutrino Observatory, Phys. Rev. Lett. 89, 011301 (2002).

[3] F. P. An et al. (Daya Bay Collaboration), Observation of Electron-Antineutrino Disappearance at Daya Bay, Phys. Rev. Lett. 108, 171803 (2012).

[4] K. Abe et al. (T2K Collaboration), First Combined Analysis of Neutrino and Antineutrino Oscillations at T2K, Phys. Rev. Lett. 118, 151801 (2017).

[5] G. G. Raffelt, Stars as Laboratories for Fundamental Physics: The Astrophysics of Neutrinos, Axions and Other Weakly Interacting Particles (University of Chicago Press, Chicago, 1996).

[6] M. Fukugita and T. Yanagida, Physics of Neutrinos and Applications to Astrophysics (Springer, Berlin, 2003).

[7] A. M. Egorov, A.E. Lobanov, and A. I. Studenikin, Neutrino oscillations in electromagnetic fields, Phys. Lett. B 491, 137 (2000).

[8] M. S. Dvornikov and A. I. Studenikin, Neutrino oscillations in the field of a linearly polarized electromagnetic wave, Phys. At. Nucl. 64, 1624 (2001).

[9] S. Meuren, C. H. Keitel, and A. Di Piazza, Nonlinear neutrino-photon interactions inside strong laser pulses, J. High Energy Phys. 06 (2015) 127.

[10] M. Formanek, S. Evans, J. Rafelski, A. Steinmetz, and C.-T. Yang, Strong fields and neutral particle magnetic moment dynamics, Plasma Phys. Controlled Fusion 60, 074006 (2018).

[11] V. G. Bagrov and D. M. Gitman, Exact Solutions of Relativistic Wave Equations (Kluwer, Dordrecht, 1990), pp. 253-257.

[12] S. R. Elliott and M. Franz, Colloquium: Majorana fermions in nuclear, particle and solid-state physics, Rev. Mod. Phys. 87, 137 (2015).

[13] M. Dvornikov, Field theory description of neutrino oscillations, in Neutrinos: Properties, Sources and Detection, edited by J. P. Greene (Nova Science Publishers, New York, 2011), pp. 23-90.
[14] C. Itzykson and J.-B. Zuber, Quantum Field Theory (McGraw-Hill, New York, 1980), pp. 691-696.

[15] L. D. Landau and E. M. Lifshitz, The Classical Theory of Fields, 4th ed. (Butterworth Heinemann, Amsterdam, 1994), p. 44.

[16] V. N. Aseev et al., An upper limit on electron antineutrino mass from Troitsk experiment, Phys. Rev. D 84, 112003 (2011).

[17] K. Abe et al. (Super-Kamiokande Collaboration), Solar neutrino measurements in Super-Kamiokande-IV, Phys. Rev. D 94, 052010 (2016).

[18] M. Dvornikov and J. Maalampi, Evolution of mixed Dirac particles interacting with an external magnetic field, Phys. Lett. B 657, 217 (2007).

[19] G. G. Likhachev and A. I. Studenikin, Neutrino oscillations in the magnetic field of the sun, supernovae, and neutron stars, J. Exp. Theor. Phys. 81, 419 (1995).

[20] M. Dvornikov, Neutrino oscillations in matter and in twisting magnetic fields, J. Phys. G 35, 025003 (2008).

[21] M. Dvornikov and J. Maalampi, Oscillations of Dirac and Majorana neutrinos in matter and magnetic field, Phys. Rev. D 79, 113015 (2009).

[22] A. G. Beda, V. B. Brudanin, V. G. Egorov, D. V. Medvedev, V.S. Pogosov, E. A. Shevchik, M. V. Shirchenko, A. S. Starostin, and I. V. Zhitnikov, Gemma experiment: The results of neutrino magnetic moment search, Phys. Part. Nucl. Lett. 10, 139 (2013).

[23] C. Y. Cardall, M. Prakash, and J. M. Lattimer, Effects of strong magnetic fields on neutron star structure, Astrophys. J. 554, 322 (2001).

[24] R. N. Manchester, Observational properties of pulsars, Science 304, 542 (2004).

[25] A. A. Danilenko, D. A. Zyuzin, Yu. A. Shibanov, and S. V. Zharikov, The Vela and Geminga pulsars in the mid-infrared, Mon. Not. R. Astron. Soc. 415, 867 (2011).

[26] T. S. Rappaport, Wireless Communications: Principles and Practice, 2nd ed. (Prentice-Hall PTR, Upper Saddle River, 2002), p. 108. 
[27] M. A. Ruderman and P. G. Sutherland, Theory of pulsars: Polar gaps, sparks, and coherent microwave radiation, Astrophys. J. 196, 51 (1975).

[28] P. Haensel, A. Y. Potekhin, and D. G. Yakovlev, Neutron Stars 1: Equation of State and Structure (Springer, New York, 2007), pp. 19-21.

[29] V. Beskin, Pulsar magnetospheres and pulsar winds, AIP Conf. Proc. 1792, 020001 (2017).
[30] A. K. Harding, Pulsar emission physics: The first fifty years, Proc. Int. Astron. Union 13, 52 (2017).

[31] M. Dvornikov and A. Studenikin, Electric charge and magnetic moment of a massive neutrino, Phys. Rev. D 69, 073001 (2004).

[32] N. F. Bell, V. Cirigliano, M. J. Ramsey-Musolf, P. Vogel, and M. B. Wise, How Magnetic is the Dirac Neutrino?, Phys. Rev. Lett. 95, 151802 (2005). 